\title{
Glucksberg Scale
}

National Cancer Institute

\section{Source}

National Cancer Institute. Glucksberg Scale. NCI Thesaurus. Code C42297.

The Glucksberg scale is a severity index for grading acute graft-versus-host disease (GVHD) after allogeneic marrow transplantation. This index is based on the results of an analysis in which distinct patterns in the peak severity of GVHD involvement in the skin, liver, and gut were correlated with the risks of transplant-related mortality (TRM) and treatment failure (defined as relapse or death). 